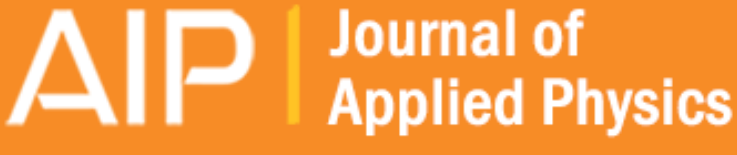

\section{A positioning-tolerant wireless charging system for roadway-powered electric vehicles}

Zhen Zhang, K. T. Chau, Chunhua Liu, Chun Qiu, and T. W. Ching

Citation: Journal of Applied Physics 117, 17B520 (2015); doi: 10.1063/1.4916187

View online: http://dx.doi.org/10.1063/1.4916187

View Table of Contents: http://scitation.aip.org/content/aip/journal/jap/117/17?ver=pdfcov

Published by the AIP Publishing

\section{Articles you may be interested in}

Effect of ferrite addition above the base ferrite on the coupling factor of wireless power transfer for vehicle applications

J. Appl. Phys. 117, 17D517 (2015); 10.1063/1.4919039

Modular inductive power transmission system for high misalignment electric vehicle application

J. Appl. Phys. 117, 17B528 (2015); 10.1063/1.4918563

Passive shielding effect on space profile of magnetic field emissions for wireless power transfer to vehicles J. Appl. Phys. 117, 17A739 (2015); 10.1063/1.4916930

Quantitative comparison of dynamic flux distribution of magnetic couplers for roadway electric vehicle wireless charging system

J. Appl. Phys. 115, 17A334 (2014); 10.1063/1.4866882

An efficient wireless power transfer system with security considerations for electric vehicle applications

J. Appl. Phys. 115, 17 A328 (2014); 10.1063/1.4866238

\section{AIP $\mid$ APL Photonics}

APL Photonics is pleased to announce Benjamin Eggleton as its Editor-in-Chief

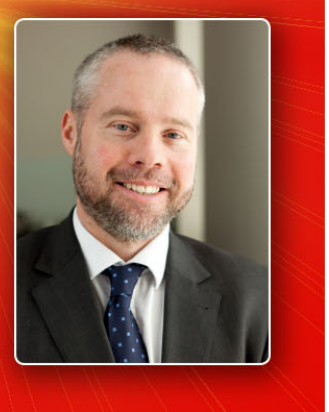




\title{
A positioning-tolerant wireless charging system for roadway-powered electric vehicles
}

\author{
Zhen Zhang, ${ }^{1, a)}$ K. T. Chau, ${ }^{1, b)}$ Chunhua Liu, ${ }^{1, c)}$ Chun Qiu, ${ }^{1}$ and T. W. Ching ${ }^{2, d)}$ \\ ${ }^{1}$ Department of Electrical \& Electronic Engineering, The University of Hong Kong, Hong Kong, China \\ ${ }^{2}$ Faculty of Science and Technology, University of Macau, Macau, China
}

(Presented 6 November 2014; received 21 September 2014; accepted 15 November 2014; published online 25 March 2015)

\begin{abstract}
This paper proposes a positioning-tolerant wireless power transfer technique to compensate the impact of misalignment on the power transmission performance, which is used to implement the wireless charging functionality in a free-positioning manner, thus significantly improving the practicality for roadway-powered electric vehicles (EVs). The key of the proposed wireless power transfer technique is to adopt the gapless alternate-winding topology for the power supply unit to produce an evenly distributed electromagnetic field and the vertical-and-horizontal coil design for the pickup unit to enhance the capability of acquiring energy. Hence, the power transmission can be effectively improved in spite of an offset between the centers of the primary and secondary coils. In this paper, both the computational simulation and experimentation are carried out to verify the feasibility of the proposed positioning-tolerant wireless charging system for roadway-powered EVs. @ 2015 AIP Publishing LLC. [http://dx.doi.org/10.1063/1.4916187]
\end{abstract}

\section{INTRODUCTION}

The roadway-powered electric vehicle (EV) can significantly alleviate the dependence on the battery. ${ }^{1}$ By utilizing the roadway powering mechanism, the EV can cordlessly obtain the energy even in the driving state. Thus, it is promising to fundamentally solve the long-term problems of EV batteries, such as their limited energy capacity, long charging time, high initial cost, heavy weight, and bulky size.

As the key of roadway powering systems, the wireless power transfer technique has increasingly attracted attentions from the academia and industry in recent years. For example, the magnetic resonant coupling scheme was utilized to increase the transmission distance for wireless power transfer systems. ${ }^{2}$ The X-rail was developed for roadway-powered EVs, which can significantly save the cost by halving the power cable use as well as activating several vehicles simultaneously and independently. ${ }^{3}$ The secure wireless power transfer scheme was proposed and implemented, which can effectively prevent unauthorized receptors from stealing the energy. ${ }^{4}$ In order to analyze the characteristics of wireless power transfer systems, an equivalent circuit model was developed via coupled magnetic resonances. ${ }^{5}$ Additionally, a comparative analysis of the circular pad with ferrite spokes and coreless rectangular coils was carried out to assess their performance for on-road EV charging. ${ }^{6}$ Also, some technical overviews were given to summarize recent works on the wireless power transmission and its application to EV battery charging. 7,8

However, there are some unsolved technical issues in the roadway powering mechanism, especially for the

\footnotetext{
${ }^{\text {a) }}$ Member, IEEE.

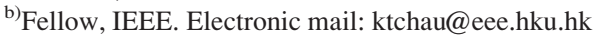

${ }^{c}$ Senior Member, IEEE.

${ }^{\text {d) }}$ Senior Member.
}

misalignment impact on the wireless charging performance. Accordingly, it is imperative to improve the misalignment tolerance between the power supply and pickup coils. By adopting the gapless alternate-winding topology for the power supply unit and the vertical-and-horizontal coil design for the pickup unit, this paper proposes and implements a positioning-tolerant roadway powering system for EVs.

\section{POSITIONING-TOLERANT WIRELESS CHARGING SYSTEM}

In this paper, the inductive power transfer technique is utilized to demonstrate the proposed positioning-tolerant wireless charging system. Fig. 1 depicts the basic circuit topology of the exemplified wireless charging system, where the involved power supply unit and pickup unit have mutual effects via the cross coupling between the primary and secondary coils. In the exemplified circuit, $C_{p}$ and $C_{s}$ are the primary and secondary capacitors, $L_{p}$ and $L_{s}$ are the inductances of the primary and secondary coils; $L_{m}$ is the mutual inductance, and $R_{p}, R_{s}$, and $R_{L}$ are the resistances of the primary, secondary, and load, respectively. Additionally, $V_{s p}$ denotes the reflected potential from the pickup unit to the power supply unit, and $V_{p s}$ denotes the reflected potential from the power supply unit to the pickup unit. Accordingly, the proposed positioning-tolerant wireless charging system will be

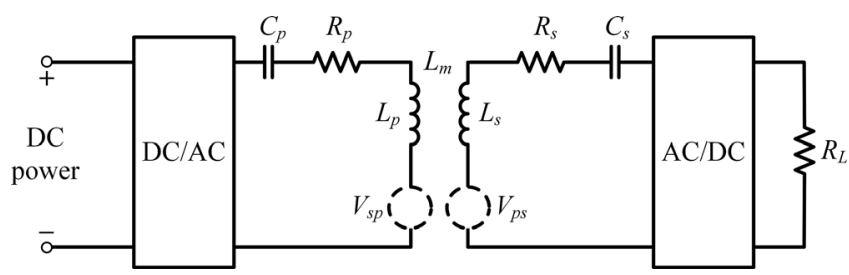

FIG. 1. Basic topology of exemplified inductive wireless power transfer system. 
TABLE I. Comparative results between same- and alternate-winding designs.

\begin{tabular}{llcc}
\hline \hline & \multicolumn{2}{c}{ Average magnetic flux density $(\mathrm{T})$} & \\
\cline { 2 - 4 } Gap (mm) & Same-winding & Alternate-winding & Sariance $\left(\mathrm{T}^{2}\right)$ \\
\hline $0 \mathrm{~mm}$ & $1.0154 \times 10^{-4}$ & $1.3659 \times 10^{-4}$ & $4.3690 \times 10^{-10}$ \\
$20 \mathrm{~mm}$ & $7.7863 \times 10^{-5}$ & $1.3791 \times 10^{-4}$ & $1.1200 \times 10^{-9}$ \\
$40 \mathrm{~mm}$ & $6.5153 \times 10^{-5}$ & $1.2208 \times 10^{-4}$ & $1.4314 \times 10^{-9}$ \\
$60 \mathrm{~mm}$ & $6.1499 \times 10^{-5}$ & $1.0457 \times 10^{-4}$ & $1.1902 \times 10^{-9}$ \\
$80 \mathrm{~mm}$ & $5.8241 \times 10^{-5}$ & $8.5820 \times 10^{-5}$ & $1.0164 \times 10^{-9}$ \\
$100 \mathrm{~mm}$ & $5.4707 \times 10^{-5}$ & $7.3371 \times 10^{-5}$ & $9.73910 \times 10^{-10}$ \\
$120 \mathrm{~mm}$ & $5.1744 \times 10^{-5}$ & $6.2275 \times 10^{-5}$ & $1.0768 \times 10^{-9}$ \\
\hline \hline
\end{tabular}

implemented by optimizing the primary and secondary coils, respectively.

\section{A. Proposed gapless alternate-winding design}

The key of the proposed positioning-tolerant wireless charging system is to produce an evenly distributed electromagnetic field by optimizing the design of primary coils. In order to comparatively illustrate various design schemes, this study utilizes the triangular packing topology to assemble 7 primary coils having the number of turns of 20 , outer diameter of $70 \mathrm{~mm}$, inner diameter of $60 \mathrm{~mm}$, and height of $8 \mathrm{~mm}$. Additionally, this study adopts two assessment indices to quantitatively analyze various design schemes, namely, the average magnetic flux density and the magnetic field distribution variance. The average magnetic flux density describes the capability of transferring energy, which is given by

$$
\bar{B}=\frac{\sum_{i=1}^{N} B_{i}}{N},
$$

where $B_{i}$ denotes the magnetic flux density of the $i$ th sample point, and $N$ denotes the number of sample points. Additionally, the magnetic field distribution variance is used to assess the distribution of the induced electromagnetic field, which is given by

$$
D=\sum_{i=1}^{N}\left(B_{i}-\bar{B}\right)^{2}
$$

In the exemplified test, the average flux density and distribution variance are calculated based on 2914 sample points on the plane of $300 \times 302.25 \mathrm{~mm}^{2}$. As shown in Table I, the comparative results show that the alternate-winding topology has higher magnetic flux density than the same-winding design, though it exhibits slightly higher variance. Accordingly, the capability of transferring energy can be effectively enhanced by adopting the alternate-winding design instead of the conventional same-winding design. Moreover, by comparing the results of the alternate-winding topology, the gapless design is the optimal scheme, which can offer approximately the maximum magnetic flux density as well as the minimum variance. Thus, the gapless alternate-winding topology is the optimal scheme for the power supply unit to enhance the capability of transferring energy and improve the distribution of the induced electromagnetic field.

\section{B. Proposed vertical-and-horizontal pickup coil}

Apart from optimizing the topology of primary coils, this paper also takes into account the design of the pickup coil to further improve the energy transmission performance. As shown in Fig. 2, the direction of magnetic lines is varying with respect to different positions. Namely, it shows a vertical line at positions above the coil and a horizontal line at positions between coils. As a result, the conventional pickup coil, namely, a single vertical coil, cannot effectively acquire the energy at the position between coils due to the horizontal magnetic line. Accordingly, the proposed design scheme installs an additional horizontal pickup coil orthogonally assembled inside the vertical pickup coil, aiming to enhance the capability of acquiring the energy at positions between coils.

Therefore, the proposed positioning-tolerant wireless charging system integrates the gapless alternate-winding primary coils and the vertical-and-horizontal secondary coil, aiming to improve the distribution of the induced electromagnetic field and enhance the capability of acquiring the energy at different positions between coils. Hence, the roadway-powered EV can effectively harness the energy in a free-positioning manner.

\section{VERIFICATION}

In this paper, a downscale prototype is utilized to validate the feasibility of the proposed positioning-tolerant wireless charging system for roadway-powered EVs, where the working frequency is chosen at $100 \mathrm{kHz}$. First, the

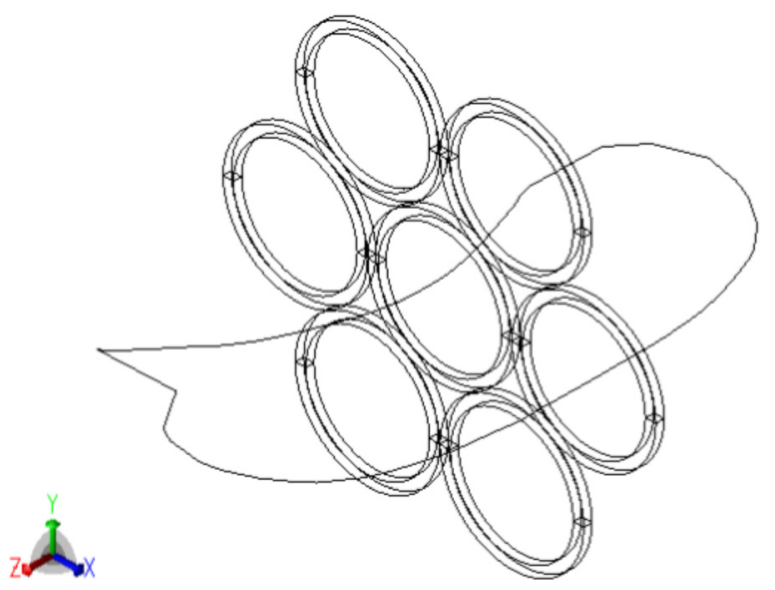

FIG. 2. Magnetic lines between coils. 


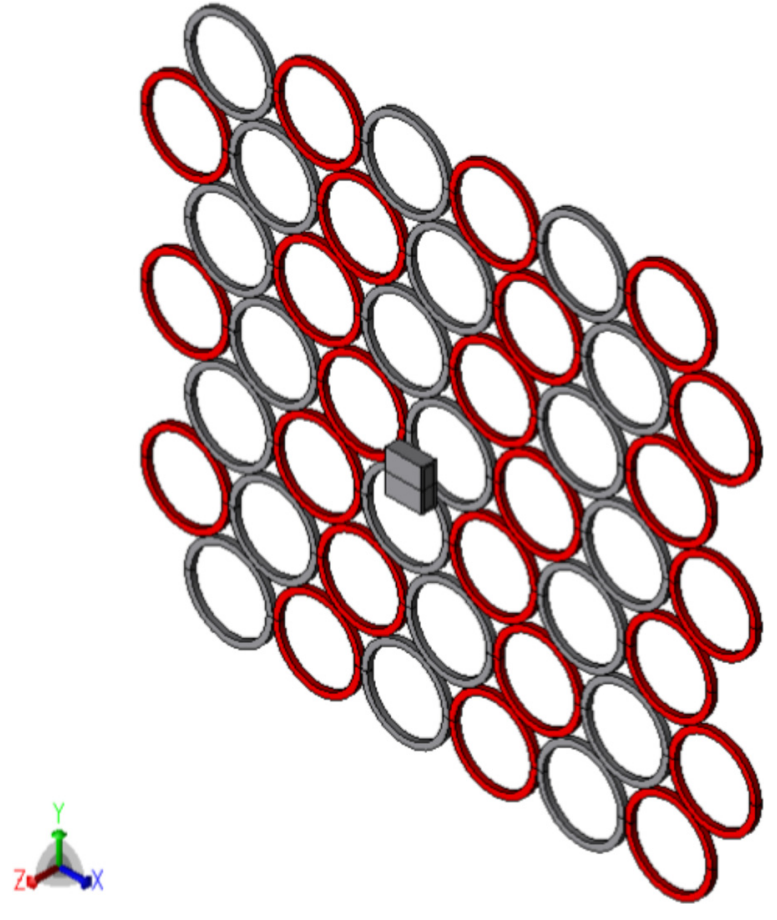

FIG. 3. Geometry of proposed primary coils.

computational simulation is conducted to illustrate the energy transmission performance and analyze the induced electromagnetic field. Then, the corresponding experimentation is carried out to verify the proposed design scheme and simulation results.

\section{A. Simulation results}

As shown in Fig. 3, the exemplified prototype adopts the proposed gapless alternate-winding design, where the red coil represents the clockwise winding direction and the grey coil represents the anticlockwise winding direction. As the power supply unit, all primary coils are packed within onesquare-meter area. The key parameters are listed in Table II. In the computational simulation, the electromagnetic field analysis is performed by using JMAG.

Fig. 4 shows the distribution of the magnetic flux density on the horizontal surface at $50 \mathrm{~mm}$ above the power supply unit. It illustrates that the magnetic flux density can be successfully maintained within a narrow range, namely, from $1.2 \times 10^{-4} \mathrm{~T}$ to $1.5 \times 10^{-4} \mathrm{~T}$. Especially for positions between coils, the proposed alternate-winding topology can effectively compensate the reduction of magnetic flux density as compared with the conventional schemes. Thus, the

TABLE II. Key parameters of exemplified wireless charging system.

\begin{tabular}{lccccc}
\hline \hline Primary coil & \multicolumn{3}{c}{ Secondary vertical coil } & \multicolumn{2}{c}{ Secondary horizontal coil } \\
\hline Outer radius & $75 \mathrm{~mm}$ & Outer width & $58 \mathrm{~mm}$ & Outer width & $65.5 \mathrm{~mm}$ \\
Inner radius & $70 \mathrm{~mm}$ & Outer length & $58 \mathrm{~mm}$ & Outer length & $65.5 \mathrm{~mm}$ \\
Height & $8 \mathrm{~mm}$ & Inner width & $58 \mathrm{~mm}$ & Inner width & $58 \mathrm{~mm}$ \\
Turn number & 20 & Inner length & $50.5 \mathrm{~mm}$ & Inner length & $58 \mathrm{~mm}$ \\
& & Height & $22.5 \mathrm{~mm}$ & Height & $22.5 \mathrm{~mm}$ \\
& & Turn number & 96 & Turn number & 138 \\
\hline \hline
\end{tabular}

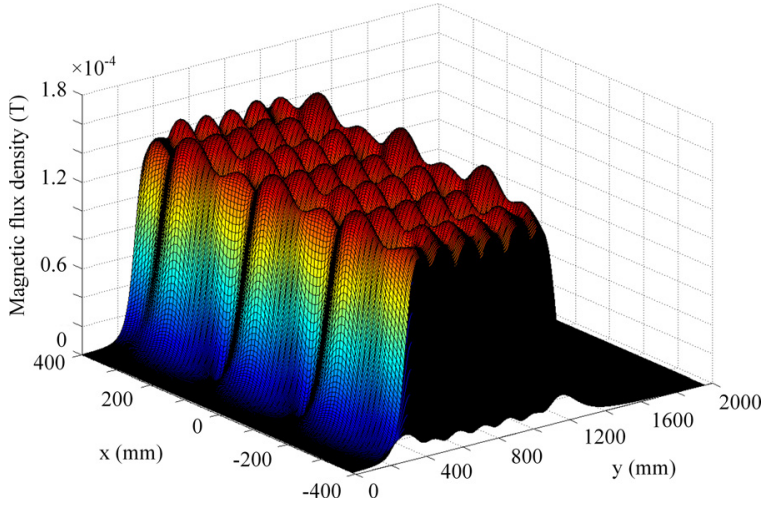

FIG. 4. Magnetic flux density distribution.

induced electromagnetic field can be evenly distributed over a wide area by using the proposed gapless alternate-windingbased coil design for the power supply unit.

Fig. 5 depicts the induced voltages by adopting the horizontal, vertical, and the proposed pickup coils, respectively. As shown in Figs. 5(a) and 5(b), the voltage value varies over a wide range when using the conventional coil design schemes, namely, from $1 \mathrm{~V}$ to $10 \mathrm{~V}$ for the horizontal pickup
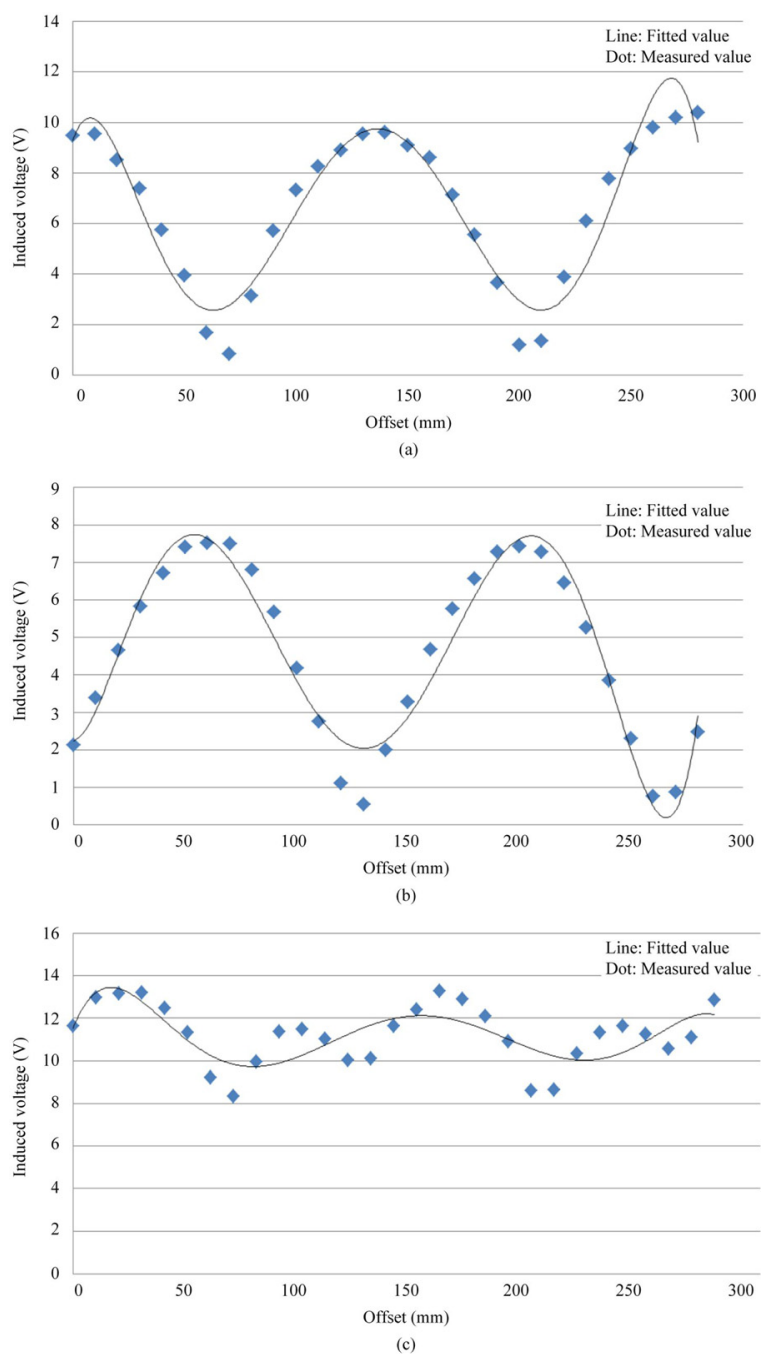

FIG. 5. Simulated induced voltages: (a) using horizontal coil; (b) using vertical coil; (c) using proposed coil. 
coil and from $1 \mathrm{~V}$ to $8 \mathrm{~V}$ for the vertical pickup coil. By adopting the proposed vertical-and-horizontal pickup coil, the variation of the induced voltage can be significantly reduced within a range of $5 \mathrm{~V}$ as shown in Fig. 5(c), namely, from $8 \mathrm{~V}$ to $13 \mathrm{~V}$. More importantly, the minimum voltage value can be successfully increased from $1 \mathrm{~V}$ to $8 \mathrm{~V}$, which ensures the receptor to constantly acquire sufficient energy, even at those gap positions between the primary and secondary coils. In addition, the transmission efficiency can be maintained at about $95 \%$. Thus, the proposed scheme can effectively offer a positioning-tolerant wireless charging system to deal with the impact of misalignment on the energy transmission performance for roadway-powered EVs.

\section{B. Experimental results}

In this paper, experimentation is also carried out to further verify the feasibility of the proposed positioningtolerant wireless power transfer system. The exemplified prototype adopts the power supply unit with the alternatewinding coil design and the secondary unit with the verticaland-horizontal coil design. All the primary and secondary coils are designed based on the same parameters with the simulation as listed in Table II.

By using the proposed vertical-and-horizontal pickup coil, this study experimentally measures the induced voltage on the area at $50 \mathrm{~mm}$ above the power supply unit. Fig. 6 shows the corresponding distribution of induced voltages, where the $x$-axis and $y$-axis denote the horizontal and vertical directions, respectively. It shows that the voltage value can reach around $15 \mathrm{~V}$ at the center of primary coils and around $6 \mathrm{~V}$ at positions between primary coils. Thus, the receptor can effectively harness sufficient energy even at misaligned positions. Additionally, the measured induced voltages are also plotted with the simulated results as shown in Fig. 5, which confirms that the experimental results well agree with the computational simulation. It further illustrates that the proposed gapless alternate-winding design for primary coils and vertical-and-horizontal secondary coil can effectively improve the distribution of the induced electromagnetic field, hence ensuring the receptor wirelessly acquire the energy in a free-positioning manner. Accordingly, the proposed position-tolerant wireless power transfer technique can successfully deal with the impact of misalignment on the energy transmission performance for roadway-powered EVs.

In addition, the proposed energy transmission system simply adopts the alternate-winding scheme to produce the evenly distributed electromagnetic field, which does not involve additional device or hardware investment, thus having no impact on the cost. Besides, an additional vertical coil is assembled in the secondary coil to enhance the capability of picking up the energy, which inevitably increases the cost.

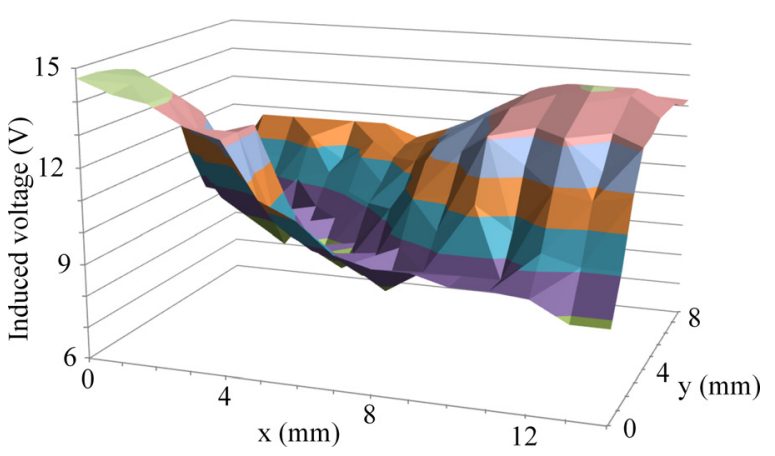

FIG. 6. Measured induced voltage distribution.

Nevertheless, for the whole system, the associated additional cost will not be significant, probably negligible.

\section{CONCLUSION}

This paper proposes and implements a positioningtolerant wireless charging technique for roadway-powered EVs, which is used to deal with the impact of misalignment on the energy transmission performance. In this study, the proposed wireless charging system adopts the gapless alternate-winding topology for the power supply unit to improve the distribution of the induced electromagnetic field, and the vertical-and-horizontal coil design for the pickup unit to enhance the capability of acquiring energy. The simulated and experimental results have verified the feasibility of the proposed positioning-tolerant wireless charging system, as well as the significant improvement on the capability of acquiring energy for roadway-powered EVs.

\section{ACKNOWLEDGMENTS}

This work was supported by a grant (Project No. HKU SPF 201109176034) from the Committee on Research and Conference Grants, The University of Hong Kong, Hong Kong.

${ }^{1}$ C. C. Chan and K. T. Chau, Modern Electric Vehicle Technology (Oxford University Press, 2001).

${ }^{2}$ A. Kurs, A. Karalis, R. Moffatt, J. D. Joannopoulos, P. Fisher, and M. Soljačić, Science 317, 83 (2007).

${ }^{3}$ S. Choi, J. Huh, W. Y. Lee, S. W. Lee, and C. T. Rim, IEEE Trans. Power Electron. 28, 12 (2013)

${ }^{4}$ Z. Zhang, K. T. Chau, C. Liu, C. Qiu, and F. Lin, J. Appl. Phys. 115, 17A328 (2014)

${ }^{5}$ S. Cheon, Y. H. Kim, S. Y. Kang, M. L. Lee, J. M. Lee, and T. Zyung, IEEE Trans. Ind. Electron. 58, 2906 (2011).

${ }^{6}$ C. Qiu, K. T. Chau, C. Liu, W. Li, and F. Lin, J. Appl. Phys. 115, 17A334 (2014).

${ }^{7}$ I. Mayordomo, T. Drager, P. Spies, J. Bernhard, and A. Pflaum, Proc. IEEE 101, 1302 (2013).

${ }^{8}$ F. Musavi and W. Eberle, IET Power Electron. 7, 60 (2014). 